\title{
A linear stochastic differential equation driven by a Fractional Brownian Motion with Hurst parameter $>\frac{1}{2}$
}

\author{
Mamadou Abdoul Diop ${ }^{\mathrm{a}, *}$, Youssef Ouknine ${ }^{\mathrm{b}}$, \\ ${ }^{a}$ Department of Mathematics, Faculty of Applied Sciences and Technology, Gaston Berger University, 234 \\ Saint-Louis, Sénégal \\ ${ }^{b}$ Department of Mathematics, Faculty of Sciences Semlalia, Cadi Ayyad University, 2390 Marrakesh, \\ Morocco
}

\begin{abstract}
Given a fractional Brownian motion $\left(B_{t}^{H}\right)_{t \geq 0}$, with Hurst parameter $>\frac{1}{2}$ we study the properties of all solutions of :

$$
X_{t}=B_{t}^{H}+\int_{0}^{t} X_{u} d \mu(u), \quad 0 \leq t \leq 1
$$

A different stochastic calculus is required for the process because it is not a semimartingale.
\end{abstract}

Keywords: Linear stochastic differential equation, Fractional Brownian motion, Stochastic calculus, Itô formula.

2000 MSC: $60 \mathrm{H} 15$

\section{Introduction}

Fractional Brownian motion (fBm) with Hurst parameter $H \in(0,1)$ is a zero mean Gaussian process $B^{H}=\left\{B_{t}^{H}, t \geq 0\right\}$ with covariance function

$$
R_{H}(s, t)=\frac{1}{2}\left(t^{2 H}+s^{2 H}-|t-s|^{2 H}\right) .
$$

This process was introduced by Kolmogorov and al (1940) and later studied by Mandelbrot and Van Ness (1968), where a stochastic integral representation in terms of a standard Brownian motion was obtained. The self similar and long range dependence (if $H>1 / 2$ ) properties of the $\mathrm{fBm}$ make this process a useful driving noise in models arising in physics, telecommunication networks, finance and other fields.

Since $B^{H}$ is not a semimartingale and it is not a Markov process if $H \neq 1 / 2$ (see Rogers (1997)), this implies that the usual stochastic calculus is not applicable for

\footnotetext{
${ }^{*}$ Corresponding author

Email addresses: mamadou-abdoul.diop@ugb.edu.sn (Mamadou Abdoul Diop), ouknine@ucam.ac.ma (Youssef Ouknine)
} 
$\left(B_{t}^{H}, t \geq 0\right)$ if $H \in\left(\frac{1}{2}, 1\right)$. In recent years some new techniques have been developed in order to define stochastic integrals with respect to $\mathrm{fBm}$.

When $H>1 / 2$, one can use a path-wise approach to define integrals with respect to the fractional Brownian motion, taking advantage of the results by Young (1936). An alternative approach to define path-wise integrals with respect to a $\mathrm{fBm}$ with parameter $H>1 / 2$ is based on fractional calculus. This approach was introduced by Feyel and De la Pradelle (1996) and it was also developed by Zähle (1998).

The aim of this paper is to describe the properties of all the continuous solutions of the following stochastic differential equation

$$
X_{t}=B_{t}^{H}+\int_{0}^{t} X_{u} d \mu(u), \quad 0 \leq t \leq 1,
$$

which is a one dimensional linear stochastic differential equation where $B_{t}^{H}$ is a fractional Brownian motion with Hurst parameter $H \in(1 / 2,1)$.

A different stochastic calculus is required. This work is inspired by that of Jeulin and Yor (1990) which corresponds to the case where $H=\frac{1}{2}$. The paper is organized as follows. In Section 2 we give the problem formulation. Section 3 contains the study of the uniqueness criterion and the existence of the solutions. In Section 4 we study the $\left(\mathcal{F}_{t}\right)$ adaptedness of the the solutions. An example is given in Section 5 and finally, in Section 6 we discuss about the time-inversion of certain diffusions and related singular equations .

\section{Problem formulation}

Initially, a fractional Brownian motion is more completely described. Let $\Omega=$ $C_{0}\left(\mathbb{R}^{+}, \mathbb{R}\right)$ be the Fréchet space of real-valued continuous functions on $\mathbb{R}^{+}$with the initial value zero and the topology of local uniform convergence. There is a probability measure, $\mathbb{P}^{H}$ on $(\Omega, \mathcal{F})$ where $\mathcal{F}$ is the Borel $\sigma$-algebra on $\Omega$ such that on the probability space $\left(\Omega, \mathcal{F}, \mathbb{P}^{H}\right)$, the coordinate process is a fractional Brownian motion, $\left(B_{t}^{H}, t \geq 0\right)$, that is,

$$
B^{H}(t, \omega)=\omega(t),
$$

for each $t \in \mathbb{R}^{+}$and (almost) all $\omega \in \Omega$. This probability space is used subsequently. Fix $H \in(1 / 2,1)$ and let $\Phi_{H}: \mathbb{R} \longrightarrow \mathbb{R}_{+}$be given by

$$
\Phi_{H}(t)=H(2 H-1)|t|^{2 H-2} .
$$

It follows by direct computation that

$$
\mathbb{E}\left[B^{H}(t) B^{H}(s)\right]=\int_{0}^{t} \int_{0}^{s} \Phi_{H}(u-v) d u d v .
$$

Let $\left(X_{t}, t \geq 0\right)$ be the $\mathbb{R}$-valued Gaussian process that is the solution of

$$
X_{t}=B_{t}^{H}+\int_{0}^{t} X_{u} d \mu(u), \quad 0 \leq t \leq 1,
$$


where $\mu$ is a Radon diffuse measure on $] 0,1]$. Our aim is to describe the properties of all the continuous solutions of (4) where

$\int_{0}^{t} X_{u} d \mu(u)$ is defined as $\lim _{\varepsilon \longrightarrow 0}$ a.s. $\int_{\varepsilon}^{t} X_{u} d \mu(u)$, limit that we suppose the existence.

To the measure $\mu$ we associate the function

$$
M(t)=\exp (\mu(] t, 1])) \quad(0<t \leq 1) .
$$

We will use recurently the fact that the process $X_{t}$ verify the relation

$$
X_{t}=X_{u} \frac{M(u)}{M(t)}+\frac{1}{M(t)} \int_{u}^{t} M(r) d B_{r}^{H}, \quad 0<u \leq t \leq 1
$$

\section{Uniqueness Criterion and existence of solutions}

\subsection{Uniqueness criterion}

We now give criterion which ensure uniqueness of the solutions.

Let $X^{1}, X^{2}$ be two solutions, and define

$$
\forall t \in[0,1], x(t)=X_{t}^{1}-X_{t}^{2} .
$$

Then $x(t)$ satisfy the following equation

$$
x(t)=\int_{0}^{t} x(r) d \mu(r)
$$

We then deduce that $x(t) M(t)$ is a constant function on $] 0,1]$. Since we must have $\lim _{t \rightarrow 0} x(t)=0$, we immediately deduce the following

Proposition 3.1. Equation(4) admits a unique solution if and only if $M(t)$ does not converge to $\infty$ when $t$ tends to zero.

If $M(t) \rightarrow \infty$ when $t \rightarrow 0$, all the solutions are deducted of one of them by the addition of $\frac{C}{M(t)}$, where $C$ is a random variable.

In particular if there is a solution, there is a unique one $X^{(1)}$, such that $X_{1}^{(1)}=0$.

\subsection{Existence of solutions}

In this section we are interested by the existence of solutions. We will discuss two cases : a priori uniqueness and non-uniqueness.

Case 1: A priori uniqueness

From proposition (3.1), we have : $\underline{\lim }_{u \rightarrow 0} M(u)<\infty$; Let $\left(u_{n}\right)$ be a sequence of real numbers, $u_{n}>0, \quad u_{n} \longrightarrow 0$ and $\underline{\lim }_{u \rightarrow 0} M(u)=\lim _{n} M\left(u_{n}\right) ;\left(X_{u_{n}} \frac{M\left(u_{n}\right)}{M(t)}\right)_{n}$ converge a.s to 0 and from (5), $X_{t}=\lim _{n} \frac{1}{M(t)} \int_{u_{n}}^{t} M(r) d B_{r}^{H}$. 
It follows from Ruzmaikina (2000) that if $\left.\left.M \in L^{\frac{2}{1+H}}(] 0,1\right]\right)$, then the limit exists in $\left.\left.L^{2}(] 0,1\right] \times \Omega\right)$ for all $\left.\left.t \in\right] 0,1\right]$ and we have :

$$
X_{t}=X_{t}^{(0)}=\frac{1}{M(t)} \int_{0}^{t} M(r) d B_{r}^{H} \quad \text { for all } t>0 ;
$$

Conversely, if $\left.\left.M \in L^{\frac{2}{1+H}}(] 0,1\right]\right)$, the process $X^{(0)}$ defined by (7) has a t-continuous version for all $t \in] 0,1]$. Apply a particular case of the Itô formula(Corollary 4.4 in Duncan and al (2000) to the process $\left.\left(X_{t}^{(0)}, 0<\varepsilon<t \leq 1\right)\right)$. Then

$$
\begin{aligned}
X_{t}^{0} & =X_{\varepsilon}^{0}+\int_{\varepsilon}^{t} d B_{r}^{H}-\int_{\varepsilon}^{t}\left(\int_{0}^{r} M(u) d B_{u}^{H}\right) \frac{d M(r)}{M^{2}(r)} \\
& =X_{\varepsilon}^{0}+B_{t}^{H}-B_{\varepsilon}^{H}-\int_{0}^{\varepsilon} X_{u}^{0} d \mu(u) .
\end{aligned}
$$

Equation. (4) admits a solution (equal to $X^{(0)}$ ) if and only if, $X_{t}^{(0)} \longrightarrow 0$ a.s; since $X^{(0)}$ is gaussian, this necessitates that $X_{t}^{(0)}$ converges to 0 in $L^{2}$ :

$$
\lim _{t \rightarrow 0} \frac{1}{M^{2}(t)} \mathbb{E}\left[\left(X_{t}^{(0)}\right)^{2}\right]=0 .
$$

Case 2: Non-uniqueness

If there exists a solution, there is a unique one $X^{(1)}$ such that $X_{1}^{(1)}=0$.

Let us note $\psi_{t}=-X_{1-t}^{(1)}$ and $\tilde{\beta}_{t}^{H}=B_{1}^{H}-B_{1-t}^{H}$. We remark that $\beta_{t}^{H}$ is a fractional Brownian motion and $\psi$ is the solution of the following equation

$$
\psi_{t}=\beta_{t}^{H}-\int_{0}^{t} \psi_{u} d \tilde{\mu}(u) \quad(t<1)
$$

where $\tilde{\mu}$ is the image of $\mu$ by $t \longrightarrow 1-t$.

This equation admits a unique solution $\left(\psi_{t}\right)_{t<1}$. The existence of a solution of the Eq.(4) will be solved if we have $\lim _{t \rightarrow 1} \psi_{t}=0$ a.s.

Since

$$
\left.\psi_{t}=\int_{0}^{t} \exp (-\tilde{\mu}(] r, t]\right) d \tilde{\beta}_{r}^{H}=\frac{1}{M(1-t)} \int_{0}^{t} M(1-r) d \tilde{\beta}_{r}^{H},
$$

$\lim _{t \rightarrow 1} \psi_{t}=0$ necessitates that

$$
\lim _{t \rightarrow 1} \frac{1}{M^{2}(1-t)} \mathbb{E}\left[\left(\int_{0}^{t} M(1-r) d \tilde{\beta}_{t}^{H}\right)^{2}\right]=0
$$

Proposition 3.2. 1. If $\underline{\lim }_{t \rightarrow 0} M(t)<\infty$, equation(4) have a solution if and only if the following conditions are satisfied:

$$
\left.\left.M \in L^{\frac{2}{1+H}}(] 0,1\right]\right) .
$$

The solution is $X_{t}^{(0)}=\frac{1}{M(t)} \int_{0}^{t} M(r) d B_{r}^{H}$. 
2. If $\lim _{t \rightarrow 0} M(t)=\infty$, there is a solution of (4) if and only if :

$$
\lim _{t \rightarrow 0} \frac{1}{M(t)} \int_{t}^{1} M(r) d B_{r}^{H}=0 .
$$

$$
\begin{aligned}
& X_{t}^{(1)}=-\frac{1}{M(t)} \int_{t}^{1} M(r) d B_{r}^{H} \text { is the solution of equation(4) such that } \\
& X_{1}^{(1)}=0 .
\end{aligned}
$$

Next, we present two lemmas concerning the stochastic integral

$$
I^{H}(t)=\int_{0}^{t} M(r) d B_{r}^{H} .
$$

The first lemma provides an upper bound for the $q$ th absolute moment of $I^{H}(t)$, while the second lemma provides a bound on the growth of the stochastic integral(11).

Lemma 3.1. For $q \geq 1$

$$
\mathbb{E}\left|I^{H}(t)\right|^{q} \leq K t^{q H}
$$

where $K$ is a constant that only depends on $q$ and $I^{H}(t)$ is given by (11).

Proof. Since, $I^{H}(t)$ is a centered Gaussian random variable, for every $q \geq 1$, there exists a constant $K_{1}$ that depends on $q$ such that

$$
\begin{aligned}
\mathbb{E}\left|I^{H}(t)\right|^{q} & \leq K_{1}(q)\left(\mathbb{E}\left(\int_{0}^{t} M(r) d B_{r}^{H}\right)^{2}\right)^{\frac{q}{2}} \\
& \leq K_{2}(q)\left(\int_{0}^{t} \int_{0}^{t} \Phi(u, v) d u d v\right)^{\frac{q}{2}} \\
& \leq K t^{q H}
\end{aligned}
$$

Lemma 3.2. For each $H \in\left(\frac{1}{2}, 1\right)$, the following equation is satisfied

$$
\lim _{t \rightarrow+\infty} \frac{\left|I^{H}(t)\right|}{t^{2 H}}=0 \quad \text { a.s. }
$$

where $I^{H}(t)$ is given by $(11)$.

Proof. Fix $n \in \mathbb{N}$ and consider the sequence of random variables $\left(I^{H}\left(\frac{k}{2^{n}}\right), \quad k \in\right.$ $\mathbb{N})$. For $k \in \mathbb{N}$, let

$$
\Delta_{k}=\left\{\frac{\left|I^{H}\left(\frac{k}{2^{n}}\right)\right|}{\left(\frac{k}{2^{n}}\right)^{2 H}} \geq 1\right\}
$$

Applying Markov's inequality for $q>1$ and (12), it follows that

$$
\begin{aligned}
\mathbb{P}\left(\Delta_{k}\right) & \leq \frac{\mathbb{E}\left|I^{H}\left(\frac{k}{2^{n}}\right)\right|^{q}}{\left(\frac{k}{2^{n}}\right)^{2 q H}} \\
& \leq K\left(\left(\frac{k}{2^{n}}\right)^{q H-2 q H}\right. \\
& \leq \tilde{K} k^{-q H},
\end{aligned}
$$


where $\tilde{K}=K .2^{-n q H}$. Since $-2 H<0$, choose $q>1$ so that

$$
\sum_{k=1}^{\infty} \frac{1}{k^{-q H}}<\infty .
$$

By the Borel-Cantelli Lemma

$$
P\left(\Delta_{k} \text { infinitely often }\right)=0
$$

Thus

$$
\lim _{k \rightarrow \infty} \sup \frac{\left|I^{H}\left(\frac{k}{2^{n}}\right)\right|}{\left(\frac{k}{2^{n}}\right)^{2 H}}=0 \quad \text { a.s. }
$$

There is a set $\Gamma$ with $P(\Gamma)=0$ such that if $\omega \in \Gamma^{c}$ then

$$
\lim _{k \rightarrow \infty} \sup \frac{\left|I^{H}\left(\frac{k}{2^{n}}, \omega\right)\right|}{\left(\frac{k}{2^{n}}\right)^{2 H}}=0
$$

for all $n \in \mathbb{N}$. Since $\left\{\frac{k}{2^{n}}, k \in \mathbb{N}\right\} \subset\left\{\frac{k}{2^{n+1}}, k \in \mathbb{N}\right\}$, it follows that $\left(\left|I^{H}(t)\right| / t^{2 H}, t \in\right.$ $D)$ converges to 0 as $t \longrightarrow \infty$, where $D=\left\{\frac{k}{2^{n}}: k, n \in \mathbb{N}\right\}$. Since $\left(\left|I^{H}(t)\right| / t^{2 H}, t \geq\right.$ $0)$ has continuous sample paths, it follows that

$$
\lim _{t \rightarrow+\infty} \frac{\left|I^{H}(t)\right|}{t^{2 H}}=0 \text { a.s. }
$$

\section{Study of the adaptedness to the filtration $\left(\mathcal{F}_{t}\right)$}

In this section we are interested by the adaptedness to $\left(\mathcal{F}_{t}\right)$. We will discuss two cases : existence and uniqueness and non uniqueness.

Case 1: Existence and uniqueness

Following proposition (3.2), the unique solution is $\left(\mathcal{F}_{t}\right)$-adapted.

Case 2: Non-uniqueness

In this case we have the following proposition

Proposition 4.1. When $\lim _{t \rightarrow 0} M(t)=\infty$, the equation (4) admits a $\left(\mathcal{F}_{t}\right)$-adapted solution if and only if :

$$
\left.\left.M \in L^{\frac{2}{1+H}}(] 0,1\right]\right)
$$

in this case, the adapted solutions are given by $X^{(0)}+\frac{C}{M}$ where $C$ is a $\mathcal{F}_{0}$-measurable random variable. 
Proof. Under the hypothesis (14), $X^{(0)}$ is a solution of $(4) ; X^{(0)}$ is $\mathcal{F}_{t}$-adapted and any other solution is obtained by adding to $X^{(0)}$ a process of the form $\frac{C}{M(t)}$; then a condition for adaptedness to $\mathcal{F}_{t}$ is that $C$ be $\mathcal{F}_{0}$-mesurable.

Conversely suppose that $X$ is an $\mathcal{F}_{t}$-adapted solution and let us show that (14) holds. From (5), we have for $0<u<t$,

$$
X_{t}=X_{u} \frac{M(u)}{M(t)}+\frac{1}{M(t)} \int_{u}^{t} M(r) d B_{r}^{H}
$$

and for a real number $\lambda$, we have by using Hölder inequality with exponent $p=\frac{1}{H}$ and $q=\frac{1}{1-H}$ that

$$
\begin{aligned}
\mathbb{E}\left[\exp \left(i \lambda X_{t}\right)\right] & =\mathbb{E}\left[\mathbb{E}\left[\exp \left(i \lambda\left(X_{u} \frac{M(u)}{M(t)}+\frac{1}{M(t)} \int_{u}^{t} M(r) d B_{r}^{H}\right)\right) \mid \mathcal{F}_{u}\right]\right] \\
& \leq\left[\mathbb{E}\left[\mathbb{E}\left(\exp \left(i \frac{\lambda}{H} X_{u} \frac{M(u)}{M(t)}\right)\right) \mid \mathcal{F}_{u}\right]\right]^{H} \times\left[\mathbb{E}\left[\mathbb{E}\left(\exp \left(i \frac{\lambda}{(1-H) M(t)} \int_{u}^{t} M(r) d B_{r}^{H}\right)\right) \mid \mathcal{F}_{u}\right]\right]^{1-H} \\
& \leq\left[\mathbb{E}\left(\exp \left(i \frac{\lambda}{H} X_{u} \frac{M(u)}{M(t)}\right)\right)\right]^{H} \times\left[\mathbb{E}\left[\mathbb{E}\left(\exp \left(i \frac{\lambda}{(1-H) M(t)} \int_{u}^{t} M(r) d B_{r}^{H}\right)\right) \mid \mathcal{F}_{u}\right]\right]^{1-H}
\end{aligned}
$$

We deduce from (15), $t>0$ fixed, and letting $u \longrightarrow 0$ :

$$
\left|\mathbb{E}\left[\exp \left(i \lambda X_{t}\right)\right]\right| \leq\left[\mathbb{E}\left(\exp \left(i \frac{\lambda}{(1-H) M(t)} \int_{0}^{t} M(r) d B_{r}^{H}\right)\right)\right]^{1-H}
$$

then we see that if condition (14) is not satisfied, then we would have for any $\lambda \neq$ $0, \mathbb{E}\left[\exp \left(i \lambda X_{t}\right)\right]=\infty$, wich is not compatible with the continuity in $\lambda=0$ of the characteristic function of the variable $X_{t}$.

\section{Example}

We consider $\mu(u)=\frac{\lambda}{u}(\lambda \neq 0)$; then $M(u)=u^{-\lambda}$.

- There is uniqueness if $\lambda<0$; then we have :

$$
\int_{0}^{1} \frac{1}{M(u)}\left(\mathbb{E}\left[\left(\int_{0}^{u} M(r) d B_{r}^{H}\right)^{2}\right]\right)^{\frac{1}{2}} d|\mu|(u)<\infty ;
$$

The solution is $X_{t}^{(0)}=t^{\lambda} \int_{0}^{t} r^{-\lambda} d B_{r}^{H}$.

- If $\lambda>0, \lim _{t \rightarrow 0} M(t)=\infty$; then

$$
\int_{0}^{1} \frac{1}{M(u)}\left(\mathbb{E}\left[\left(\int_{u}^{1} M(r) d B_{r}^{H}\right)^{2}\right]\right)^{\frac{1}{2}} d|\mu|(u)<\infty ;
$$

The solutions $X_{t}^{\lambda}=C t^{\lambda}+t^{\lambda} \int_{0}^{t} r^{-\lambda} d B_{r}^{H}$ are continuous in 0 . $X_{t}^{(1)}=-t^{\lambda} \int_{0}^{t} r^{-\lambda} d B_{r}^{H}$. 


\section{Time-inversion of certain diffusions, and related singular equations.}

\subsection{Some singular equations}

We are interested in the following singular stochastic differential equation :

$$
X_{t}=x+B_{t}^{H}+\int_{0}^{t} b\left(u, X_{u}\right) d u, \quad t \geq 0
$$

where the function $b(s, x)$ has a singularity at $s=0$.

We now show how to associate, to certain diffusions $\left(X_{t}, t \geq 0\right)$ which are "canonical" solutions of (16) a singular equation analogous to

$$
X_{t}=\beta_{t}^{H}+2 H \int_{0}^{t} \frac{X_{s}}{s} d s, \quad t \geq 0,
$$

using time-inversion.

Here, we assume that the process $\left(X_{t}\right)$ is adapted to the natural filtration of $\left(B_{t}^{H}\right)$, and that the Eq.(16) has only one strong solution.

Now, let $0<s<t$. We have :

$$
\frac{X_{t}}{t^{2 H}}=\frac{X_{s}}{s^{2 H}}-2 H \int_{s}^{t} \frac{X_{u}}{u^{2 H+1}} d u+\int_{s}^{t} \frac{b\left(u, X_{u}\right)}{u^{2 H}} d u+\int_{s}^{t} \frac{d B_{u}^{H}}{u^{2 H}} .
$$

We now assume that :

$$
\lim _{t \rightarrow+\infty} \frac{X_{t}}{t^{2 H}} \rightarrow 0 \text { and moreover }, \lim _{t \rightarrow+\infty} \int_{s}^{t} \frac{X_{u}}{u^{2 H+1}} d u \text { exists a.s. }
$$

Then, letting $t \rightarrow \infty$ in (18), we see that, since $\lim _{t \rightarrow+\infty} \int_{1}^{t} \frac{d B_{u}^{H}}{u^{2 H}}$ exists a.s., the limit :

$$
\lim _{t \rightarrow+\infty} \int_{1}^{t} \frac{b\left(u, X_{u}\right)}{u^{2 H}} d u \text { also exists. }
$$

Hence, we deduce from (18) and (19) that :

$$
0=\frac{X_{s}}{s^{2 H}}-2 H \int_{s}^{\infty} \frac{X_{u}}{u^{2 H+1}} d u+\int_{s}^{\infty} \frac{b\left(u, X_{u}\right)}{u^{2 H}} d u+\int_{s}^{\infty} \frac{d B_{u}^{H}}{u^{2 H}} .
$$

Now, we take $s=\frac{1}{t}$, and define,$\hat{X}_{t}^{H}=t^{2 H} X_{\frac{1}{t}}$; we remark that

$$
\begin{gathered}
\beta_{t}^{H}=-\int_{\frac{1}{t}}^{\infty} \frac{d B_{u}^{H}}{u^{2 H}}, t>0 \text { is a fractional Brownian motion. Then, we obtain : } \\
\hat{X}_{t}^{H}=\beta_{t}^{H}+2 H \int_{0}^{t} \frac{\hat{X}_{v}^{H}}{v^{2 H}} d v-\int_{0}^{t} b\left(\frac{1}{v}, \frac{\hat{X}_{v}^{H}}{v^{2 H}}\right) \frac{v^{2 H}}{v^{2}} d v
\end{gathered}
$$

In the particular case $b \equiv 0$, we recover the equality (17): indeed, $\left(X_{t}^{H}, t \geq 0\right)$ is a fractional Brownian motion; hence, in this case, (20) tell us that :

$$
\left(\hat{X}_{t}^{H}-2 H \int_{0}^{t} \frac{\hat{X}_{v}^{H}}{v} d v \quad t \geq 0\right) \text { is a fractional Brownian motion. }
$$




\subsection{Resolution of some singular equations}

We would like to find all solutions of the following equations $\left(E_{k}^{H}\right)$ and $\left(E_{A}^{H}\right)$ :

$$
\left(E_{k}^{H}\right) \quad X_{t}=\gamma_{t}^{H}+2 H \int_{0}^{t} \frac{X_{s}}{s} d s-k \int_{0}^{t} \frac{s^{2 H-1}}{X_{s}} d s
$$

where, here, $\left(X_{t}\right)_{t \geq 0}$ is only assumed to be a continuous process, valued in $\mathbb{R}^{+}, k>$ 0 , and both integrals $\int_{0}^{t} \frac{X_{s}}{s} d s$ and $\int_{0}^{t} \frac{s^{2 H-1}}{X_{s}} d s$ converge ; $\left(\gamma_{t}^{H}, t \geq 0\right)$ is a fractional Brownian motion starting from 0 ;

$$
\left(E_{l}^{H}\right) \quad X_{t}=\gamma_{t}^{H}+2 H \int_{0}^{t} \frac{X_{s}}{s} d s-l_{t}^{H} .
$$

Again, here, $\left(X_{t}, t \geq 0\right)$ is only assumed to be a continuous process, valued in $\mathbb{R}^{+}, \int_{0}^{t} \frac{X_{s}}{s} d s<$ $\infty,\left(l_{t}^{H}, t \geq 0\right)$ is an increasing process which only increases on the zero set of $X$.

In fact, in order not to repeat similar arguments to solve equations $\left(E_{k}^{H}\right)$, and then $\left(E_{l}^{H}\right)$, we shall first consider a more general equation :

$$
\left(E_{A}^{H}\right) \quad X_{t}=\gamma_{t}^{H}+2 H \int_{0}^{t} \frac{X_{s}}{s} d s-A_{t}^{H} \quad, t \geq 0,
$$

where the only difference with $\left(E_{k}^{H}\right)$ and $\left(E_{l}^{H}\right)$ is that, here, $\left(A_{t}^{H}, t \geq 0\right)$ is only assumed to be a continuous increasing process.

Then, we have the following preparatory

Lemma 6.1. $\left(X_{t}, t \geq 0\right)$ solves $\left(E_{A}^{H}\right)$ if and only if:

(i) $\lim _{t \rightarrow+\infty} \frac{X_{t}}{t^{2 H}}=Y^{H}$ and, if we denote : $\hat{X}_{t}^{H}=t^{2 H} X_{\frac{1}{t}}$, then this process satisfies :

(ii) $\hat{X}_{t}^{H}=Y^{H}+B_{t}^{H}+\int_{\frac{1}{t}}^{\infty} \frac{d A_{u}}{u^{2 H}}$, where : $B_{t}^{H}=-\int_{\frac{1}{t}}^{\infty}\left(\frac{d \gamma_{u}}{u^{2 H}}\right)$.

Proof. Starting from the Eq. $\left(E_{A}^{H}\right)$, we obtain, for $0<s<t$ :

$$
\frac{X_{t}}{t^{2 H}}=\frac{X_{s}}{s^{2 H}}+\int_{s}^{t} \frac{d \gamma_{u}^{H}}{u^{2 H}}-\int_{s}^{t} \frac{d A_{u}^{H}}{u^{2 H}} .
$$

Hence ,

$$
\frac{X_{t}}{t^{2 H}}+\int_{s}^{t} \frac{d A_{u}^{H}}{u^{2 H}}=\frac{X_{s}}{s^{2 H}}+\int_{s}^{t} \frac{d \gamma_{u}^{H}}{u^{2 H}}
$$

Fix $s>0$; letting $t \rightarrow \infty$, we obtain, since : $\lim _{t \rightarrow+\infty} \int_{s}^{t} \frac{d \gamma_{u}^{H}}{u^{2 H}}$ exists, that

$$
\int_{s}^{\infty} \frac{d A_{u}^{H}}{u^{2 H}}<\infty \text {, and, therefore, } \frac{X_{t}}{t^{2 H}} \text { converges as } t \rightarrow \infty \text {. Define } Y^{H}=\lim _{t \rightarrow+\infty} \frac{X_{t}}{t^{2 H}} \text {. }
$$


Now we deduce, from (21), that :

$$
\frac{X_{s}}{s^{2 H}}=Y^{H}-\int_{s}^{\infty} \frac{d \gamma_{u}^{H}}{u^{2 H}}+\int_{s}^{\infty} \frac{d A_{u}^{H}}{u^{2 H}},
$$

from which the lemma follows.

Theorem 6.1. 1. Let $k>0$. Then, $\left(X_{t}, t \geq 0\right)$ is a solution of $\left(E_{k}^{H}\right)$ if and only if it may be written in the following form .

If $B_{t}^{H}=-\int_{\frac{1}{t}}^{\infty} \frac{d \gamma_{u}^{H}}{u^{2 H}}$, and, if, for $\rho \geq 0,\left(R_{t}(\rho), t \geq 0\right)$ denotes the unique solution of

$$
Z_{t}=\rho+B_{t}^{H}+\int_{0}^{t} \frac{k d s}{Z_{s}}, \quad \text { with }: \quad Z_{s} \geq 0,
$$

then, there exists a random variable $Y^{H} \geq 0$ such that

$$
t^{2 H} X_{\frac{1}{t}}=R_{t}\left(Y^{H}\right), \quad t \geq 0 .
$$

2. A process $\left(X_{t}, t \geq 0\right)$ is a solution of $\left(E_{l}^{H}\right)$ if and only if it may be written in the following form.

Let $B_{t}^{H}=-\int_{\frac{1}{t}}^{\infty} \frac{d \gamma_{u}^{H}}{u^{2 H}}$, and, denote, for $\rho \geq 0$, by,$\left(R_{t}(\rho), t \geq 0\right)$ the unique solution of

$$
Z_{t}=\rho+B_{t}^{H}+\lambda_{t}^{H}, \quad t \geq 0, \quad \text { with } Z_{t} \geq 0,
$$

and $\left(\lambda_{t}^{H}, t \geq 0\right)$ a continuous increasing process, wich only increases on the zeros of $\left(Z_{t}, t \geq 0\right)$, then, there exists a random variable $Y^{H} \geq 0$ such that

$$
t^{2 H} X_{\frac{1}{t}}=R_{t}\left(Y^{H}\right), \quad t \geq 0 .
$$

Proof. 1) The uniqueness of the solution of (22) and (23), without assuming adaptedness is due respectively to Kean (1969) and Skorokhod (1965).

From the lemma, we obtain immediately that, if $\left(X_{t}\right)$ is a solution of $\left(E_{k}^{H}\right)$, then

$$
\hat{X}_{t}^{H}=Y^{H}+B_{t}^{H}+\int_{0}^{t} \frac{k u^{2 H-1}}{\hat{X}_{u}^{H}} d u .
$$

Then, using Mc Kean's argument, we know, on one hand, that the Eq.(24)

admits only one solution, and, on the other hand, the process $\left(R_{t}(\rho), t \geq 0\right)$ may be chosen to be jointly continuous ; hence, it follows that $\left(R_{t}\left(Y^{H}\right) ; t \geq 0\right)$ is a welldefined process which solves (24); this proves the first assertion, using again the uniqueness of the solutions of (24).

2) The proof of the second assertion is very similar.

Acknowledgments. This research was done while the author was visiting the University of Marrakech. The author gratefully acknowledges support from the Gaston Berger University specially PA2PR Project and UMMISCO. The author is greatly indebted to Foundation Hassan II and to Professor Youssef Ouknine for drawing her attention . 


\section{References}

Jeulin, T. and Yor, M.(1990), Filtration des ponts browniens et équations différentielles stochastiques linéaires, Séminaire de probabilités de Strasbourg,24, 227-265.

Duncan, T. E., Y.Z. Hu and B. Pasik-Duncan (2000), Stochastic calculus for fractional Brownian motion.I, SIAM J. Control Optim.,38, pp. 582-612.

H.P. Mc. Kean (1989), Stochastic integrals . Academic Press.

Skorokhod, A.V., (1965), Studies in the theory of random processes, Addison-Wesley

A. A. Ruzmaikina,(2000), Stieltjes integrals of Hölder continuous functions with applications to fractional Brownian motion, J. Stat. Phys, 100, 1049-1069.

Feyel, D. and de la Pradelle, A.(1996), Fractional integrals and Brownian processes, Potential Analysis, 10,273-288.

Kolmogorov, A. N.(1940), Wienersche Spiralen und einige andere interessante Kurven im Hilbertschen Raum, C. R. (Doklady) Acad. URSS (N.S.)26, 115-118.

Mandelbrot, B. B. and Van Ness, J. W.(1968), Fractional Brownian motions, fractional noises and applications, SIAM Review 10(4), 422-437.

Rogers, L. C. G. (1997), Arbitrage with fractional Brownian motion, Math. Finance 7 , 95-105.

Samko, S. G., Kilbas, A. A. and Marichev, O. I.(1993), Fractional Integrals and Derivatives. Theory and Applications. Gordon and Breach.

Young, L. C. (1936), An inequality of the Hölder type connected with Stieltjes integration, Acta Math, 67, 251-282.

Zähle, M(1998), Integration with respect to fractal functions and stochastic calculus.I, Prob. Theory Relat. Fields, 111, 333-374. 